\title{
Re-evaluation of a food frequency questionnaire to estimate total $n-3$ fatty acid intake in primary school children
}

\author{
I. Paciarotti and J. McKenzie \\ Queen Margaret University, Edinburgh EH21 6UU, UK
}

Long-chain omega-3 PUFA (LC $n$-3 PUFA), including DHA and EPA, contribute significantly to neurological and cognitive development in children ${ }^{(1)}$. Dietary sources of intake of LC $n-3$ PUFA, however, are limited only to relatively few and less commonly eaten foods and there are currently no data available on LC $n$-3 PUFA intakes in primary-aged school children. Recent studies have shown that endogenous synthesis of LC $n$-3 PUFA from $\alpha$-linolenic acid (ALA), which is abundant in the diet, is not as efficient as previously thought ${ }^{(2)}$. This casts doubt on the adequacy of the supply of LC $n-3$ PUFA based on the conversion of ALA. In response to this evidence, food manufacturers have developed several foods fortified with 'omega-3 fats' marketed at parents by suggesting benefits to children's learning and behaviour. There is currently no validated method of collecting intake data. A FFQ ${ }^{2}$ was previously design to measure the $n-3$ PUFA intake in children with the breakdown of each single $n-3$ PUFA. However, the FFQ was found to underestimate ALA intake.

The aim of this study was to re-design the previous FFQ to overcome the main limitation of ALA underestimation and then re-evaluate it. Thirty-nine primary school children (16 males and 23 female), aged 5-8 years participated in the study.

A comparison with the National Diet and Nutrition Survey ${ }^{(3)}$ showed that intakes of total $n$-3 PUFA for 5-6-year-old males $(P=0.77)$, 5-7-year-old females $(P=0.66)$, and 7-8-year-old males $(P=0.79)$ were in line with the expected frequencies, whereas intakes in 7-8year-old females were significantly lower $(P<0.001)$. Data for total $n$-3 PUFA $(P<0.001)$, ALA $(P<0.001)$, DHA $(P<0.001)$, were significantly greater compared to data from the previous study ${ }^{(4)}$, whereas intakes for and total $n-3$ LC PUFA $(P=0.5)$ and EPA $(P=0.22)$ were not significantly different. The mean intakes of total LC $n-3$ PUFA in both male $(0.18 \pm 0.005 \mathrm{~g} / \mathrm{d})$ and female $(0.18 \pm 0.04 \mathrm{~g} / \mathrm{d})$ groups, were significantly lower $(P<0.001)$ than recommendations ${ }^{(5)}$. 'Omega-3'-fortified foods did not account for any of the total $n-3$ LC PUFA intake; however, 3 children ( 1 male, 2 female) took omega- 3 supplements which accounted for $21 \%$ of the total $n$-3 LC PUFA intake from all children.

The study indicates that the FFQ was successful in overriding the main limitation of the original design, which involved underestimation of ALA intake. Furthermore, it illustrated that intakes of LC $n$-3 PUFA were far below that of recommendations. Validation of the FFQ, against a more comprehensive method of assessing dietary intake, is now fundamental to assess its accuracy.

1. Richardson AJ (2004) Long-chain polyunsaturated fatty acids in childhood developmental and psychiatric disorders. Lipids 39, $1215-1222$.

2. Burdge GC, Jones AE \& Wooton SA (2002) Eicosapentanoic and docosapentanoic acids are the principal products of $\alpha$-linolenic acid metabolism in young men. Br J Nutr 88, 355-363.

3. Gregory JR, Lowe S, Bates CJ et al. (2000) National Diet and Nutrition Survey: Young People Aged 4-18 Years.Volume 1: Report of the Diet and Nutrition Survey. London: The Stationary Office.

4. McGregor A (2008) Design of a food frequency questionnaire to measure $n$-3 fatty acids intake in children. Master Thesis, Queen Margaret University.

5. Scientific Advisory Commission on Nutrition and Committee on Toxicology (SACN/COT) (2004). Advice on Fish Consumption: Benefits and Risks. London: The Stationary Office. 